\title{
An Energy Scheduling Algorithm for Ensuring the Pre-determined Lifetime in Sensor Network
}

\author{
Yong-Man Cho, Seung-Jae Lee, Changhwa Kim, and Sangkyung Kim \\ Department of Computer Science \& Engineering, Kangnung National University \\ Gangneung Daehangno 120, Gangneung-Si, Gangwon-Do, 210-702, Korea \\ Tel.: +82-33-640-2897; Fax: +82-33-640-2899 \\ \{ymcho, silveree, kch, skkim98\}@kangnung.ac.kr
}

\begin{abstract}
Energy limitation of a sensor network is a very important feature which distinguishes it from traditional networks and a number of methods to saving energy consumption have been studied in sensor networks. Most of those works have focused on Network, MAC or PHY layer and the lifetime of a sensor network cannot be pre-determined. But some sensor network might be able to survive for some specified time. In this work, we propose an energy consumption scheduling model, at application level of a node, that ensures predetermined lifetime. To do this, first, we divide applications into six classes and define five operations of an application. Second, an energy consumption model is proposed using those five operations and consumption energies of each operation are calculated by a statistical method. Finally, energy consumption schedules of each application are rebuilt for a node to survive for predetermined lifetime, e.g. varying the period of response and sensing and the number of actuation, etc. Consequently, it is ensured for nodes to survive for the pre-determined lifetime.
\end{abstract}

Keywords: energy scheduling algorithm, pre-determined lifetime, energy consumption model.

\section{Introduction}

Recently, the progress of wireless communication and computing technology has led the development of very small-sized sensor nodes. A sensor node is composed of sensors, which gather various environmental data, sensed-data processing unit, short-ranged wireless communication transceiver, and power unit [1]. A sensor network might be considered as a distributed computing platform that has the restricted processing power, small-sized memory, and the limited bandwidth and power.

More than anything else, a sensor node's limited energy provided by a battery requires a big effort to overcome in sensor networks. Many researches have been studied focusing on the methodologies and algorithms to consume a sensor node's energy efficiently. They include energy-efficient routing, data aggregation employing 
clustering, energy-efficient MAC algorithm, data reporting period control, and so on. [2] and [3] proposed to extend the lifetime of a sensor network by minimizing energy consumption with cutting off a sensor node's transceiver while it is not participating in communications. These researches are related to enhancing energy efficiency in a MAC layer. Many researchers have studied energy-efficient routing algorithms. MMRE [5] selects a route toward the most energy-remained nodes after referring to each node's remained energy recorded in routing tables. MTE [6] selects a route consuming little energy by calculating the energy to transmit data to the next node. [7] and [8] combined the above two algorithms and suggested optimal routing algorithms. [9] and [10] control the network topology to operate minimum number of nodes within a network management area, alternately sleep nodes of which regions are overlapped to sense events, or minimize operating time by repeating sleep/wake based on each node's time table.

These researches are related to the methods to reduce the energy consumption at a whole network level and show quite good results at an entire sensor network system's perspective. While the existing researches have aimed at increasing the lifetime of a sensor network, our research intends to pre-determine the lifetime of a sensor network and make an energy consumption schedule to survive for the time. This energy consumption schedule is not a centralized, but an individual node based approach. This approach will prevent the generation of additional messages in a sensor network to mitigate the waste of energy.

The rest of this paper is as follows. Section 2 describes the basic strategy for energy consumption model. Section 3 explains our energy consumption model and scheduling algorithm. The last section remarks our conclusions.

\section{Basic Strategy for Energy Consumption Model}

To construct a energy consumption planning model, it needs the basic strategy like Fig. 1. We assume that the amount of consumption energy for an application is different from that of another application and we divide application types by

Identifies application class \& operations of it

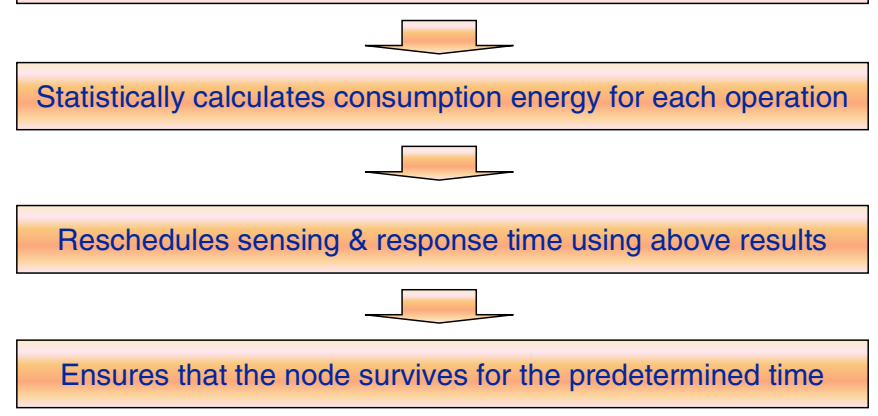

Fig. 1. Strategies for Energy Consumption Model 
characteristic of its transmission cycle and sensing cycle. As applications execute, each application consumes different amount of energy and these states could be presented with some operations. We can calculate consumption energy for each application by the operations, we make each sensor node be active until predetermined life time by changing the calculated cycle.

The structures of sensor network are divided into two categories such as unidirectional and bi-directional. In uni-directional structure, a control center cannot control sensor nodes and sensing and transmission cycle are set initially at sensor nodes. In contrast to this, in bi-directional structure, a control center can control sensor nodes and sensor nodes work with the command such as synchronization message, clustering message, routing message, data transfer message and query message.

A query (application) which is transmitted from a control center to a sensor node could be divided into 6 types by the transmission (response) cycle and the sensing cycle like below.

- Cyclic query(it does not have a pre-determined life time)

- Timed query(it does not have a transmission cycle)

- Event driven query(it has not a transmission cycle but a pre-determined life time)

- Instant query(it does not have both a transmission cycle and a life time)

- Cyclic and Timed query

- Timed event driven query

Energy consumption state variations of an application as time goes by are presented in Fig. 2.with the operations listed below.

- Transmission or Response: This operation is for sending sensed data from a sensor node to a sink node, a control center and a neighbor node on routing path. In this operation, the transmitter consumes most of energy and the processing cost for transferring a message to the network layer, the MAC layer and the PHY layer is included in this operation.

- Reception: This operation is for receiving a query at a node. In this operation, the receiver consumes most of energy and the processing cost for delivering a message to the application layer is included in this operation. This operation cannot be controlled by the node itself.

- Sensing: This operation is for transforming sensed data to information. In this operation, most of energy is consumed with sensing and delivering to a processing module. The processing cost including calibration is included in this operation.

- Processing: This operation is for processing energy consuming scheduling according to the type of applications.

- Actuation: This operation is for operating actuators. In this operation, most of energy is consumed by LEDs and motors. 


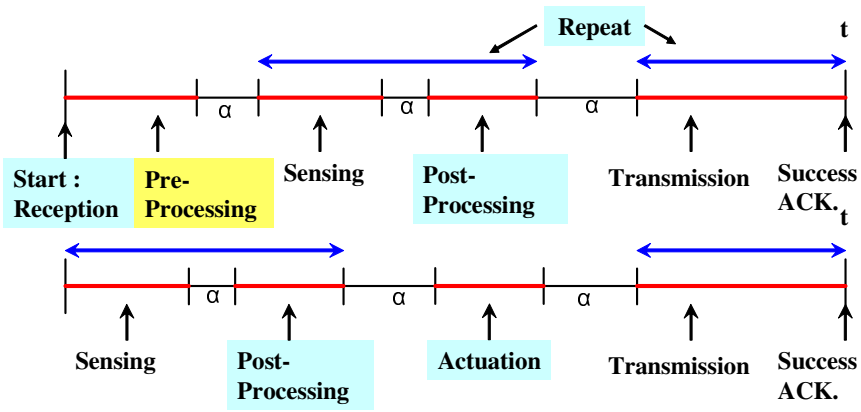

Pre-processing : Identifying the type of the application (query).

Post-processing : Verifying a sensed value and performing other operation.

Repeat : Sensing and Transmission might executable multiple times in this range

a : Terms of time in which state of an applications is undetermined.

Energy consumption might be occurred or not in a.

Fig. 2. The operation definitions for energy consumption state of a sensor node in which an application is running

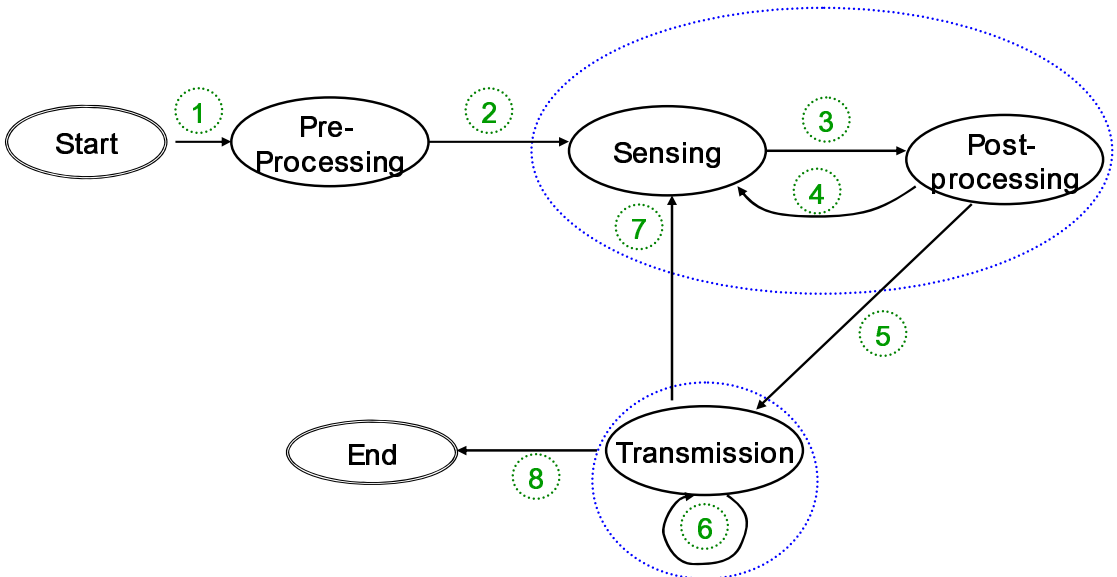

Fig. 3. State transition diagram of an application (STD: State Transition Diagram)

Fig. 3 shows the state transition diagram (STD) of an application, and explanations for each transition are like below.

(1) Prior to execute a query, it transits to Pre-processing state to analysis the type of a query.

(2) Transition to Sensing state with the type of a query. 
(3) Transition to Post-processing state with a sensed result.

(4) According to the information at Pre-processing and Sensing, whether an event occurs or not it transits to Transmission state or Sensing state if it is an event driven query. And then, according the result whether there is an error or not, it transits to Sensing state or Transmission state.

(6) Self-transition by transmission error.

(7) Transition to Sensing state on valid query execution time.

(8) Transition to End state on fired query execution time.

In Fig. 3, Actuation operation is not included. The reason is that most of sensor networks powers independently to actuators as it takes high energy.

Next, the energy model for determining consumed energy with the operations defined above is described.

\section{Energy Consumption Model and Schedule Algorithm}

An energy model is required to calculate the amount consumption energy of a node. We can express consumption energy as a function of time like (1).

$$
E=E(t)
$$

Among 5 operations defined previously, 3 operations except the transmission and sensing operations is uncontrollable. We call consumed energy by these three operations to. Therefore, we can express (1) as (2) which is a function of consumption energy of transmission and sensing.

$$
E=E_{\beta}+n_{T} \times E_{T}+n_{S} \times E_{S}
$$

$n_{T}$ : the transmission count during time t.

$n_{S}:$ the sensing count during time t.

To schedule these operations for surviving a pre-determined life time using (2), it needs (3), (4), (5) and (6).

$$
\begin{gathered}
N_{T S}=\left(E_{R E M}-\left(T_{R E M} \div T_{U N I T}\right) \times E_{\beta} \div E_{T S}\right. \\
T_{c y c l e}=T_{R E M} \div N_{T S} \\
N_{S}=\left(E_{R E M}-\left(T_{R E M} \div T_{U N I T}\right) \times E_{\beta} \div E_{S}\right. \\
N_{\text {cycle }}=T_{R E M} \div N_{S}
\end{gathered}
$$


Table 1. Parameter used in functions

\begin{tabular}{ll}
\hline Parameter & \multicolumn{1}{c}{ Description } \\
\hline$E_{T S}$ & $\begin{array}{l}\text { An estimated amount of consumption } \\
\text { energy for a transmission. }\end{array}$ \\
$E_{S}$ & $\begin{array}{l}\text { An estimated amount of consumption } \\
\text { energy for a sensing. } \\
\text { Remained time for a sensor node to be } \\
T_{R E M}\end{array}$ \\
$T_{\text {cycle }}$ & survived in future. \\
$S_{\text {cycle }}$ & Transmission cycle. \\
$N T_{S}$ & Sensing cycle. \\
$N_{S}$ & A maximum transmissible count for \\
& remained life time. \\
& A maximum sensible count for remained \\
\hline
\end{tabular}

(3) is for that sensing cycle is dependent on transmission cycle and (5) is for that transmission cycle is dependent on sensing cycle. $T_{\text {cycle }}$ and $S_{\text {cycle }}$ mean how frequently sensing and transmitting for remained lifetime. Therefore, new transmission and sensing cycle for ensuring remained lifetime can be calculated. (3) and (5) can be reduced like below, where the unit of $T_{R E M}$ is equal to $T_{U N I T}$

$$
\begin{gathered}
N_{T S}=\left(E_{R E M}-\left(T_{R E M} \times E_{\beta}\right)\right) \div E_{T S} \\
T_{\text {cycle }}=T_{R E M} \div N_{T S} \\
N_{S}=\left(E_{R E M}-\left(T_{R E M} \times E_{\beta}\right)\right) \div E_{S} \\
N_{\text {cycle }}=T_{R E M} \div N_{S}
\end{gathered}
$$

Energy consumption algorithm is for describing how to determine the counts of transmission and sensing operations for a sensor node to be survived during predetermined life time. And this could be presented with 4 steps like below.

- Step 1: Identifying a received query.

- Step 2: Applying a proper energy consumption model to the query.

- Step 3: Executing the query by a calculated transmission cycle and sensing cycle.

- Step 4: Repeating step 2 and step 3 periodically until pre-determined life time.

\section{Conclusion}

In this paper, we propose an energy consumption scheduling algorithm in a sensor node with pre-determined life time, which is different from the other works. And we 
classify application types in application layer, define the operations which consume energy and show that consumption energy by the operations can be calculated statistically. In addition, as the proposed algorithm forces that a sensor node survives for pre-determined life time, it also force that a whole sensor network survives for a given period.

Finally, research for enhancing the reliability of the algorithm proposed in this paper will be explored in the future.

Acknowledgement. This research was supported by the MIC (Ministry of Information and Communication), Korea, under the 2006 ITRC (Information Technology Research Center) contract number IITA-2006-C1090-0603-0044 support program supervised by the IITA (Institute of Information Technology Assessment).

\section{References}

1. Akyildiz, I.F., Su, W., Sankarasubramaniam, Y., Cayirci, E.: A Survey on Sensor Networks. IEEE Communications Magazine (2002)

2. Dam, T.V., Langendoen, K.: An adaptive energy-efficient MAC protocol for wireless sensor networks. In: ACM SenSys 2003 (November 2003)

3. Deb, B., Bhatnagar, S., Nath, B.: A Topology Discovery Algorithm for Sensor Networks with Applications to Network Management. Technical Report DCS-TR-441, Department of Computer Science, Rutgers University (May 2001), (submitted for publication)

4. Chang, J.-H., Tassiulas, L.: Maximum lifetime routing in wireless sensor networks. In: Presented at the ATIRP Conf, College Park, MD (March 2000)

5. Gomez, J., Campbell, A.T., Naghshineh, M., Bisdikian, C.: Power-aware routing in wireless packet networks. In: Mobile Multimedia Communication (MOMUC), San Diego (November 1999)

6. Xu, Y., Heidemann, J., Estrin, D.: Geography-informed Energy Conservation for Ad Hoc Routing. In: Mobile Computing and Networking (MobiCom), Rome (July 2001)

7. Toh, C.K.: Maximum battery life routing to support ubiquitous mobile computing in wireless ad hoc networks. IEEE communication magagine, 138-147 (June 2001)

8. Ramanathan, R., Hain, R.: Topology control of multihop wireless networks using transmit power adjustment. INFOCOM (2000)

9. Kawadia, V., Kumar, P.R.: Power control and clustering in ad hoc networks. In INFOCOM (2003) 